\title{
EVOLUTION OF INTERSTELLAR ICES
}

\author{
LOUIS J. ALLAMANDOLA, MAX P. BERNSTEIN, SCOTT A. SANDFORD and \\ ROBERT L. WALKER \\ Astrochemistry Laboratory, NASA Ames Research Center, MS 245-6, Mountain View, CA \\ 94035-1000, USA
}

\begin{abstract}
Infrared observations, combined with realistic laboratory simulations, have revolutionized our understanding of interstellar ice and dust, the building blocks of comets. Ices in molecular clouds are dominated by the very simple molecules $\mathrm{H}_{2} \mathrm{O}, \mathrm{CH}_{3} \mathrm{OH}, \mathrm{NH}_{3}, \mathrm{CO}, \mathrm{CO}_{2}$, and probably $\mathrm{H}_{2} \mathrm{CO}$ and $\mathrm{H}_{2}$. More complex species including nitriles, ketones, and esters are also present, but at lower concentrations. The evidence for these, as well as the abundant, carbon-rich, interstellar, polycyclic aromatic hydrocarbons (PAHs) is reviewed. Other possible contributors to the interstellar/pre-cometary ice composition include accretion of gas-phase molecules and in situ photochemical processing. By virtue of their low abundance, accretion of simple gas-phase species is shown to be the least important of the processes considered in determining ice composition. On the other hand, photochemical processing does play an important role in driving dust evolution and the composition of minor species. Ultraviolet photolysis of realistic laboratory analogs readily produces $\mathrm{H}_{2}, \mathrm{H}_{2} \mathrm{CO}, \mathrm{CO}_{2}, \mathrm{CO}, \mathrm{CH}_{4}, \mathrm{HCO}$, and the moderately complex organic molecules: $\mathrm{CH}_{3} \mathrm{CH}_{2} \mathrm{OH}$ (ethanol), $\mathrm{HC}(=\mathrm{O}) \mathrm{NH}_{2}$ (formamide), $\mathrm{CH}_{3} \mathrm{C}(=\mathrm{O}) \mathrm{NH}_{2}$ (acetamide), $\mathrm{R}-\mathrm{CN}$ (nitriles), and hexamethylenetetramine (HMT, $\mathrm{C}_{6} \mathrm{H}_{12} \mathrm{~N}_{4}$ ), as well as more complex species including amides, ketones, and polyoxymethylenes (POMs). Inclusion of PAHs in the ices produces many species similar to those found in meteorites including aromatic alcohols, quinones and ethers. Photon assisted PAHice deuterium exchange also occurs. All of these species are readily formed and are therefore likely cometary constituents.
\end{abstract}

\section{Interstellar ice}

Interstellar gas and dust, including ices and ice-and organic-mantled refractory dust grains, comprise the primary stuff from which the solar system formed. It is important to understand interstellar ice composition and photochemistry since these ices are considered important cometary building blocks. Furthermore, they contain more material and are more chemically complex than the interstellar gas-phase material.

Gaseous species readily condense onto cold, $10 \mathrm{~K}$ grains in dense molecular clouds forming ice mantles. Interestingly, mantle composition does not reflect gas phase composition or abundances. Differences in relative sublimation rates and chemical reactivities, complicated by photochemical processing, produce ice compositions strikingly different from the gas. New compounds are formed when reactive gaseous species condense on the grain surfaces and the ices are energetically processed by UV radiation and cosmic rays (cf. Greenberg and Li, 1999, elsewhere

Space Science Reviews 90: 219-232, 1999.

(c) 1999 Kluwer Academic Publishers. Printed in the Netherlands. 


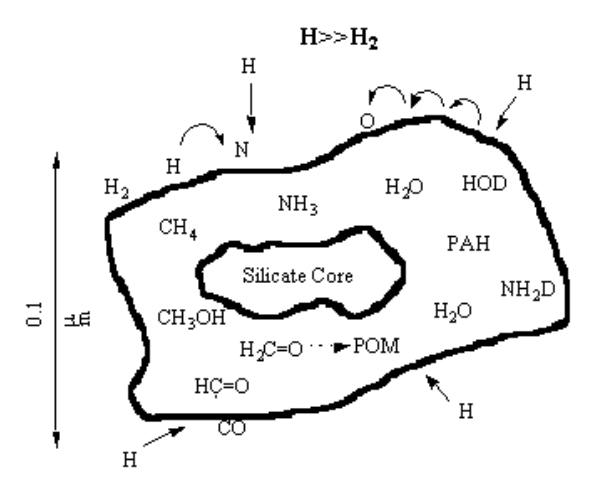

WTTHOUT ENERGETIC ENERGETIC PROCESSING ICE MANTLES
WILLBE MADE UP MOLECULES

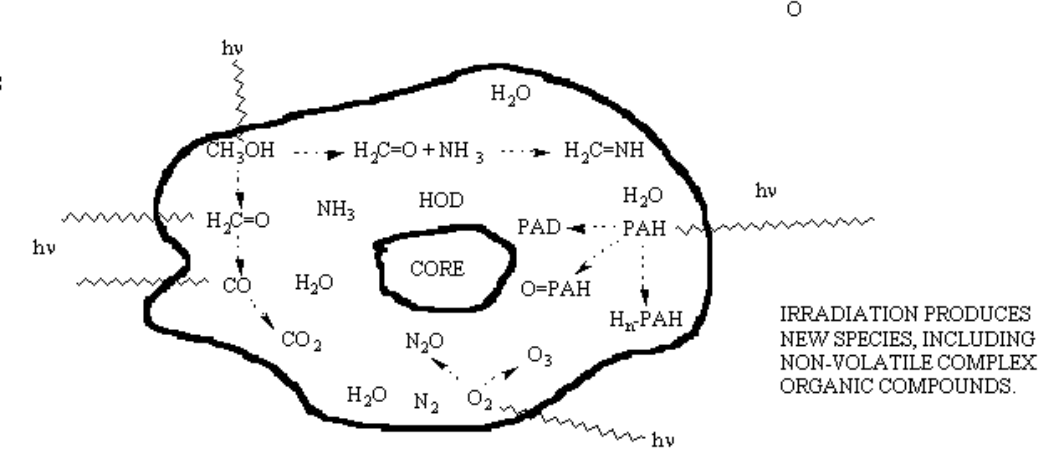

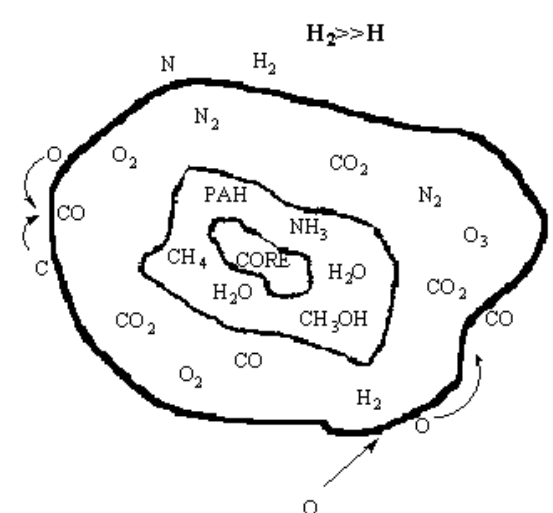
ON-VOLATILE COMPLEX

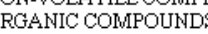

Figure 1. Schematic drawings of the types of ice mantles expected in dense molecular clouds. In regions where the $\mathrm{H} / \mathrm{H}_{2}$ ratio is much greater than one (top left), surface reactions tend to be reducing and favor the production of simple hydrides of the cosmicly abundant $\mathrm{O}, \mathrm{C}$, and $\mathrm{N}$. In contrast, oxidized forms of these species are favored in regions where this ratio is much less than one (top right). Irradiation and thermal processing (bottom) of these ice mantles creates considerably more complex species and, ultimately, non-volatile residues.

in this book; Greenberg et al., 1972; Tielens and Hagen, 1982; d'Hendecourt et al., 1985; Brown and Charnley, 1990; Moore et al., 1983; Bernstein et al., 1995). Some of these processes are schematically shown in Figure 1.

Since hydrogen is 3 to 4 orders of magnitude more abundant than the next most abundant reactive heavier elements such as $\mathrm{C}, \mathrm{N}$, and $\mathrm{O}$, overall grain surface chemistry is moderated by the $\mathrm{H} / \mathrm{H}_{2}$ ratio. In regions where this ratio is large, $\mathrm{H}$ atom addition (hydrogenation) dominates and species such as $\mathrm{H}_{2} \mathrm{O}, \mathrm{NH}_{3}$, and $\mathrm{CH}_{4}$ are expected to be prominent. If the $\mathrm{H} / \mathrm{H}_{2}$ ratio is substantially less than one, however, reactive species such as $\mathrm{O}$ and $\mathrm{N}$ are free to interact with one another forming molecules such as $\mathrm{CO}, \mathrm{CO}_{2}, \mathrm{O}_{2}$, and $\mathrm{N}_{2}$. Thus, two qualitatively different types of ice mantle are expected to be produced by grain surface reactions, one dominated by polar, H-bonded molecules and the other dominated by nonpolar, or only slightly polar, highly unsaturated molecules. Figure 1 also shows the first generation of products one might expect upon photolysis (UV irradiation) of 
these mantles. This picture of interstellar grain mantle formation and evolution is supported by the observational evidence summarized below.

Interstellar ice compositions are revealed through their infrared (IR) spectra. A star situated in or behind a molecular cloud can generate a continuous IR emission spectrum. As this radiation passes through the cloud, the intervening molecules in the gas and dust absorb at their characteristic frequencies. Since ice features tend to dominate such spectra, interstellar ice composition can be readily analyzed by making spectral comparisons with ices prepared in a laboratory under realistic interstellar conditions. Such simulations are carried out at NASA Ames and elsewhere in high-vacuum chambers where thin layers of mixed- molecular ices, comparable in thickness to those of the mantles on interstellar grains $(\sim 0.05 \mu \mathrm{m})$, are frozen on cold substrates. In a typical experiment, the spectrum of the sample is measured before and after several periods of exposure to UV radiation and thermal cycling. These spectra are then compared directly with interstellar spectra in order to identify ice composition, determine molecular abundances, and probe ice evolution. Detailed descriptions of the approach can be found in Allamandola and Sandford (1988), and Bernstein et al. (1995).

Figure 2 shows a comparison between the spectrum of W33A, a protostar embedded in a molecular cloud (Willner, 1977; Capps et al., 1978) with the laboratory spectra of interstellar ice analogs. The excellent matches between the interstellar absorption features with laboratory absorption spectra, as illustrated in Figure 2, represent the basis of our knowledge of interstellar ices. Interestingly, until quite recently, more was known of interstellar ice composition-grains hundreds of light years away-than of cometary ices in our own Solar System! A brief summary of the major components follows.

$\mathbf{H}_{2} \mathbf{O}$ (water) - $\mathrm{H}_{2} \mathrm{O}$ is the dominant ice component in dense clouds. At present, five interstellar features have been detected which fit reasonably well with laboratory $\mathrm{H}_{2} \mathrm{O}$ ice spectra (c.f. Leger et al., 1983; Tielens et al., 1984; Smith et al., 1989, 1993; Omont et al., 1990). The $3280 \mathrm{~cm}^{-1}(3.07 \mu \mathrm{m})$ band is typically one of the strongest in the interstellar spectra.

$\mathrm{CH}_{3} \mathrm{OH}$ (methanol) - Molecular cloud spectra often contain a prominent absorption near $1460 \mathrm{~cm}^{-1}(6.85 \mu \mathrm{m})$ as shown in Figure 2. It was suggested early on that much of this absorption might be due to the $\mathrm{CH}$ deformation mode vibration of methanol (Hagen et al., 1980; Tielens et al., 1984). Although supported by laboratory studies, the unequivocal identification of methanol required the detection of its other bands (Grim et al., 1991; Allamandola et al., 1992). $\mathrm{CH}_{3} \mathrm{OH}$ abundances deduced from all of the features taken together suggest that other species contribute to the interstellar absorption around $1460 \mathrm{~cm}^{-1}$. Aliphatic organic compounds and carbonates are reasonable candidates (cf. Allamandola and Sandford, 1988).Gas 


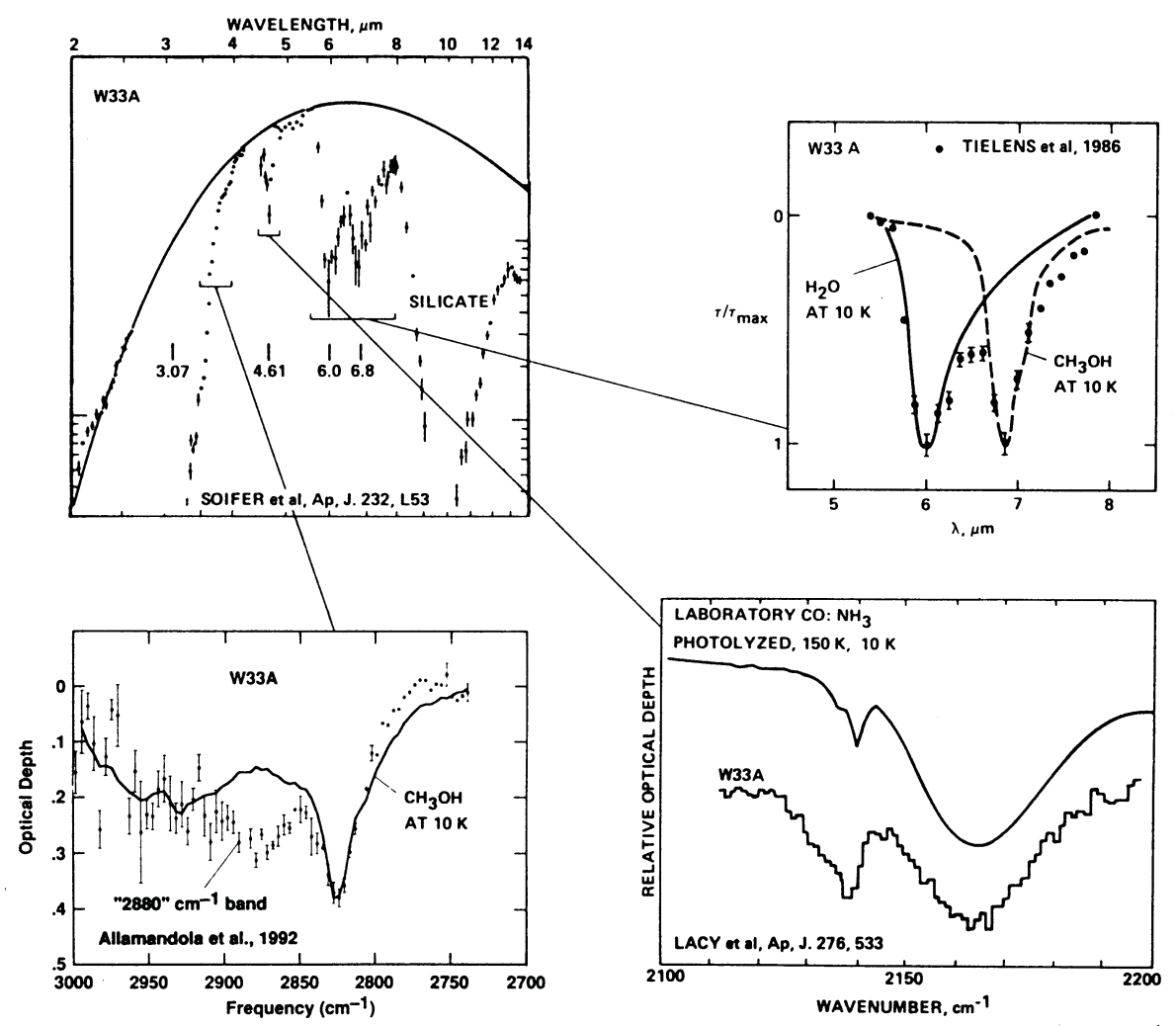

Figure 2. Comparisons of laboratory analog spectra with spectra from the object W33A, a protostar deeply embedded in a dense molecular cloud. Upper left- The dots trace out the interstellar spectrum and the solid line corresponds to the quasi-blackbody emission spectrum thought to be produced by the protostar. The strong absorption near $10 \mu \mathrm{m}$ is due to the silicate grains in the cloud, and the excess absorption labeled "2880" is thought to arise from interstellar microdiamonds. The other absorptions are produced by interstellar ices. These bands are presented on expanded scales and compared to laboratory spectra in the surrounding frames. Lower left- The solid line is due to methanol in a laboratory ice. Lower right- The upper smooth line corresponds to a laboratory analog comprised of $\mathrm{CO}$ (sharp band) and XCN (broad band). Upper right- The solid and dashed lines correspond to spectra of $\mathrm{H}_{2} \mathrm{O}$ and $\mathrm{CH}_{3} \mathrm{OH}$ respectively.

phase methanol enhancements have been found in star and planet forming regions of dense clouds where the $\mathrm{CH}_{3} \mathrm{OH}$ is thought to be liberated from warming ices.

CO (carbon monoxide) - After $\mathrm{H}_{2} \mathrm{O}$, the most studied interstellar ice component is probably carbon monoxide. $\mathrm{CO}$ has a characteristic absorption feature near $2140 \mathrm{~cm}^{-1}(4.67 \mu \mathrm{m})$, as shown in Figure 2. Its position, width, and profile are a sensitive function of the ice matrix in which the $\mathrm{CO}$ is frozen (Sandford et al., 1988; Elsila et al., 1997). Many, but not all, of the lines-of-sight that contain $\mathrm{H}_{2} \mathrm{O}$ 
ice also contain $\mathrm{CO}$ ice and the relative strengths of the $\mathrm{H}_{2} \mathrm{O}$ and $\mathrm{CO}$ bands indicate $\mathrm{CO} / \mathrm{H}_{2} \mathrm{O}$ ratios ranging from 0.0 to as much as 0.3 (Lacy et al., 1984; Tielens et al., 1991; Chiar et al., 1994, 1995, 1998). Although a few of the CO bands have positions and profiles consistent with $\mathrm{CO}$ frozen in $\mathrm{H}_{2} \mathrm{O}$-rich matrices, most linesof-sight exhibit profiles indicative of $\mathrm{CO}$ frozen in both non-polar matrices, i.e., ices thought to be dominated by molecules such as $\mathrm{CO}, \mathrm{CO}_{2}, \mathrm{O}_{2}, \mathrm{~N}_{2}$, and $\mathrm{CH}_{4}$; and polar, $\mathrm{H}_{2} \mathrm{O}$ dominated matrices. These are the two sorts of mantle predicted on the basis of the $\mathrm{H} / \mathrm{H}_{2}$ ratio discussed earlier and sketched in Figure 1. The fact that $\mathrm{H}_{2} \mathrm{O}$, a highly polar molecule, is the most abundant molecule in the ices along all these lines-of-sight, but that the $\mathrm{CO}$ is generally in ices dominated by nonpolar molecules provides clear evidence for the existence of multiple chemical environments within individual clouds. Elsila et al. (1997) and Ehrenfreund et al. $(1996,1997)$ have shown that these non-polar ices are likely to be dominated by $\mathrm{N}_{2}, \mathrm{O}_{2}$, and $\mathrm{CO}_{2}$. In the more quiescent regions as much as $40 \%$ of the cosmic $\mathrm{N}$ and $25 \%$ of the cosmic $\mathrm{O}$ could be in the form of frozen $\mathrm{N}_{2}$ and $\mathrm{O}_{2}$. If comets indeed contain unmodified interstellar ices, these highly volatile species could drive cometary activity at large heliocentric distances.

"XCN" - The spectra of a limited number of lines-of-sight through dense clouds contain a broad, often weak, feature near $2165 \mathrm{~cm}^{-1}(4.62 \mu \mathrm{m})$ (Figure 2; Lacy et al., 1984; Tegler et al., 1993, 1995). While the statistics are currently poor, there is an indication that this feature is present, or at least prominent, only in the spectra of objects embedded within clouds which are associated with protostellar and protoplanetary regions and not in the spectra of background stars (Tegler et al., 1995). This suggests that, as with methanol, the band carrier is associated with the local environment of the embedded star. Laboratory experiments show that the interstellar band can be reproduced by photolyzing ice mixtures containing $\mathrm{C}$ and N (Lacy et al., 1984; Tegler et al., 1993; Bernstein et al., 1995). This, plus the frequency, implicate the $\mathrm{C} \equiv \mathrm{N}$ functional group in a larger molecule, and suggest that some form of energetic processing is needed to produce it in the interstellar medium. Isotope and ion trapping experiments make a good case for assigning this feature to $\mathrm{OCN}^{-}$(Grim and Greenberg, 1987a; Schutte and Greenberg, 1997; Demyk et al., 1998).

$\mathbf{H}_{2}$ (hydrogen) - Molecular hydrogen $\left(\mathrm{H}_{2}\right)$ may have been detected along the line-of-sight to WL5 in the $\rho$ Ophiucus cloud (Sandford et al., 1993). This is a deeply embedded object which produces gas-phase $\mathrm{CO}$ bands which overlap the possible $\mathrm{H}_{2}$ band. Confirmation awaits high resolution spectroscopy. In any event, laboratory studies of interstellar ice analogs show that ion and UV irradiation produce, and efficiently trap, frozen $\mathrm{H}_{2}$ (Moore and Hudson, 1992; Sandford and Allamandola, 1993b). This process can be very efficient, producing $\mathrm{H}_{2}$ abundances up to one third of the $\mathrm{H}_{2} \mathrm{O}$. Thus, hydrogen could have a high abundance in interstellar and cometary ices. 
TABLE I

Composition and abundances of interstellar ice (relative to $\mathrm{H}_{2} \mathrm{O}$ ) compared to that deduced in Comets Halley, Hyakutake, and Hale-Bopp. The species listed above HCO in Column Two have been definitely detected, those below have been tentatively identified. The evidence is good for all of these species. Cometary abundances are from: Halley - Altwegg et al. (1999); Hyakutake and Hale-Bopp - Crovisier and Bockelée-Morvan (1999). See text for interstellar ice references.

\begin{tabular}{lcrrr}
\hline MOLECULE & $\begin{array}{c}\text { INTERSTELLAR ICE } \\
\text { Abundance }\end{array}$ & \multicolumn{3}{c}{ COMET PARENT MOLECULES } \\
Halley & Hyakutake & Hale-Bopp \\
\hline $\mathrm{H}_{2} \mathrm{O}$ & 100 & 100 & 100 & 100 \\
$\mathrm{CO}$ (polar ice) & $1-10$ & & & \\
$\mathrm{CO}$ (non-polar ice) & $10-40$ & 17 & $6-30$ & 20 \\
$\mathrm{CH}_{3} \mathrm{OH}$ & $<4-10$ & 1.25 & 2 & 2 \\
$\mathrm{CO}_{2}$ & $1-10$ & 3.5 & $<7$ & 6 \\
$\mathrm{XCN}$ & $1-10$ & - & - & - \\
$\mathrm{NH}_{3}$ & $5-10$ & 1.5 & 0.5 & 0.7 \\
$\mathrm{H}_{2}$ & $\sim 1$ & - & - & - \\
$\mathrm{CH}_{4}$ & $\sim 1$ & $<0.8$ & 0.7 & 0.6 \\
$\mathrm{HCO}$ & $\sim 1$ & - & - & - \\
$\mathrm{H}_{2} \mathrm{CO}$ & $1-4$ & 3.8 & $0.2-1$ & 1 \\
$\mathrm{~N}_{2}$ & $10-40$ & - & - & - \\
$\mathrm{O}_{2}$ & $10-40$ & - & - & - \\
$\mathrm{OCS}_{2}$ or $\mathrm{CO}_{3}$ & few & 0.2 & 0.1 & 0.3 \\
\hline
\end{tabular}

Other Species - Based on limited telescopic observations, laboratory studies of ice analogs, and theoretical chemistry models, a number of other molecular species are suspected of being present in interstellar ices in quantities on the order of a few percent relative to $\mathrm{H}_{2} \mathrm{O}$. Until recently, the following species have been tentatively identified in small numbers of objects: $\mathrm{CH}_{4}$ (methane, Lacy et al., 1991), $\mathrm{CO}_{2}$ (carbon dioxide, d'Hendecourt and Jordain de Muizon, 1989) $\mathrm{HCO}$ and $\mathrm{H}_{2} \mathrm{CO}$ (formyl radical and formaldehyde, cf. Tielens and Allamandola, 1987; Schutte et al., 1996), OCS (carbonyl sulfide, Palumbo et al., 1995) or $\mathrm{CO}_{3}$ (carbon trioxide, Elsila et al., 1997), $\mathrm{N}_{2}$ and $\mathrm{O}_{2}$ (Elsila et al., 1997; Ehrenfreund et al., 1996, 1997), and possibly ketones and/or aldehydes (Tielens and Allamandola, 1987). The diatomic species $\mathrm{S}_{2}$, of relevance to comets as it has been detected in a cometary coma, is also readily made upon irradiation of an interstellar ice analog (Grim and Greenberg, 1987b).

Interstellar $\mathrm{NH}_{3}$ ice has now been definitely detected, with a concentration between 5 and 10\% that of the water (Lacy et al., 1998). Recent IR spectral observations by the ISO satellite have shown that frozen $\mathrm{CO}_{2}$ is ubiquitous in dense 
clouds, having an abundance varying between roughly 10 and $15 \%$ of the $\mathrm{H}_{2} \mathrm{O}$, and that $\mathrm{CH}_{4}$ is also a common interstellar ice component, typically at the few percent level (DeGrauw et al., 1996; Boogert et al., 1997). Over the next few years, ISO spectra will certainly deepen our understanding of interstellar ice. These interstellar ice constituents and their average abundances are compared to those of comets in Table I.

\section{Gas Phase Accretion and Gas-grain Chemistry}

Of course, any gas phase species observed in dense clouds by radio (or infrared) techniques should also be present in the grain mantles (e.g. Irvine, 1999; Winnewisser and Kramer, 1999). At the low temperatures characteristic of these environments, all molecules should be strongly depleted by condensation onto the ice (cf. Sandford and Allamandola, 1993b). This includes the polycyclic aromatic hydrocarbons (PAHs) which are known, through their infrared emission, to be ubiquitous and abundant gas phase species throughout the interstellar medium (cf. Allamandola et al., 1989; Puget and Leger, 1989; Brook et al., 1999). However, as shown in Table II, the much lower abundances of the specific gas-phase molecules known from radio observations require that direct accretion of most complex gas-phase species plays a very minor role in determining interstellar ice composition. This relationship also holds for the radio-quiet $\mathrm{CH}_{4}$ as shown by recent ISO observations (Boogert et al., 1997).

As mentioned earlier, the solid CO spectral characteristics along lines-of-sight which probe quiescent portions of molecular clouds can be quite distinct from those closely associated with embedded protostars. The narrow CO band that is characteristic of non-polar ices seems to be associated with lines-of-sight which sample the colder, quiescent regions of dense clouds.

This is consistent with the picture that the ices in the protostellar environment have probably undergone substantially more radiative processing and are somewhat warmer (i.e., above $30 \mathrm{~K}$ ) than those ices along lines of sight which sample quiescent regions. Since the protostellar environment is also the formation site of new planetary systems, the interstellar/precometary ice composition in these regions is of particular relevance to comets. These are the regions in which the XCN and $\mathrm{CH}_{3} \mathrm{OH}$ bands are prominent, suggesting that these materials are associated more with the radiation-rich and somewhat warmer environment of the protostar than with the molecular cloud itself. It is now clear that $\mathrm{CH}_{3} \mathrm{OH}$ is often the second or third most abundant component in these and cometary ices (cf. Reuter, 1992; Allamandola et al., 1992). The high abundance of methanol is of key importance since its presence drives a rich interstellar photochemistry (Allamandola et al., 1988; Bernstein et al., 1995) and gas phase chemistry (Charnley et al., 1992). Furthermore, since methanol has profound effects on the physical behavior of $\mathrm{H}_{2} \mathrm{O}$-rich ices, this may have important implications for their structural (Blake 
TABLE II

Comparison between the gas-phase and solid-state abundances for several molecular species normalized to hydrogen. This comparison shows that the interstellar ices contain the bulk of interstellar polyatomic molecules.

\begin{tabular}{|c|c|c|c|c|}
\hline \multicolumn{5}{|c|}{ MOLECULAR ABUNDANCE WITH RESPECT TO HYDROGEN } \\
\hline Molecule & $\begin{array}{c}\text { Gas phase } \\
\text { TMC-1 }\end{array}$ & $\begin{array}{c}\text { Gas phase } \\
\text { OMC-1 }\end{array}$ & $\begin{array}{c}\text { Ice } \\
\text { NGC } 7538 \text { IR9 }\end{array}$ & $\begin{array}{c}\text { Ice/Gas } \\
\text { Ratio }\end{array}$ \\
\hline $\mathrm{CO}$ & $8 \times 10^{-5}$ & $5 \times 10^{-5}$ & $6 \times 10^{-6}$ & 0.12 \\
\hline $\mathrm{H}_{2} \mathrm{O}$ & - & - & $6 \times 10^{-5}$ & $?$ \\
\hline $\mathrm{CH}_{3} \mathrm{OH}$ & $2 \times 10^{-9}$ & $1 \times 10^{-7}$ & $6 \times 10^{-6}$ & $60-3000$ \\
\hline $\mathrm{NH}_{3}$ & $2 \times 10^{-8}$ & - & $6 \times 10^{-6}$ & 300 \\
\hline $\mathrm{CO}_{2}$ & $5 \times 10^{-8}$ & - & $8 \times 10^{-6}$ & 160 \\
\hline $\mathrm{CH}_{4}$ & - & - & $6 \times 10^{-7}$ & $?$ \\
\hline $\begin{array}{l}\text { Gas Phase } \\
\mathrm{CO}_{2}-\text { van } \\
\text { Ice mantle } \\
\mathrm{CH}_{4}-\mathrm{Bo}\end{array}$ & $\begin{array}{l}\text { les: TMC-1 } \\
\text { hoek et al. } \\
\text { lamandola } \\
\text { et al. (1997 }\end{array}$ & $\begin{array}{l}\text { hishi et al. ( } \\
\text { (1992); CO }\end{array}$ & $\begin{array}{l}\text { OMC-1 - Blake } \\
\text { eGrauw et al. }\end{array}$ & 87); \\
\hline
\end{tabular}

et al., 1991; Ehrenfreund et al., 1998) and vaporization behavior (Sandford and Allamandola, 1993b).

Thorough recent reviews on interstellar ices can be found in Sandford et al. (1996) and Schutte (1996).

\section{Interstellar Ice Evolution}

As discussed earlier, the ices in dense molecular clouds are irradiated by UV photons and cosmic rays, breaking and rearranging chemical bonds within the ice. This is an important process since it can create complex molecular species that cannot be made via gas phase and gas-grain reactions at the low temperatures and pressures characteristic of dense clouds. The reason for this is that solid-phase reaction kinetics, stoichiometry, and energetics favor complexity and chemical diversity.

As an example, for the last three pages we consider the photochemical evolution of an interstellar ice analog comprised of $\mathrm{H}_{2} \mathrm{O}: \mathrm{CH}_{3} \mathrm{OH}: \mathrm{CO}: \mathrm{NH}_{3}$ (100:50:10:10). Exposure to UV destroys several species (particularly methanol) and creates others such as: $\mathrm{HCO}, \mathrm{H}_{2} \mathrm{CO}, \mathrm{CH}_{4}, \mathrm{CO}_{2}$, XCN, etc. (Allamandola et al., 1988; Bernstein et al., 1995), all of which have been identified in interstellar ices (Table I). Presently, the strongest evidence that radiation processing is important, at least in some locations within dense clouds, is provided by the "XCN" feature (Figure 2), which 
Species evident at $200 \mathrm{~K}$<smiles>CCOCCCCCCCCC=O</smiles><smiles>CC(N)=O</smiles>

Species remaining at $300 \mathrm{~K}$

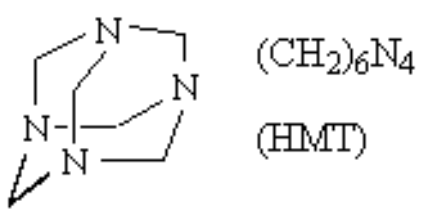<smiles>[R]C(O)C(N)ON</smiles>

$\mathrm{CH}_{3} \mathrm{CH}_{2}-\mathrm{OH}$ (ethanol)<smiles>[R]C([R])=O</smiles>

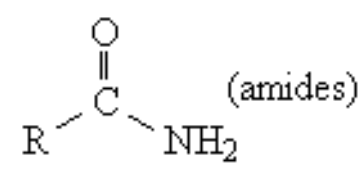

Figure 3. Compounds produced by the $10 \mathrm{~K} \mathrm{UV}$ photolysis of the realistic interstellar ice analogs $\mathrm{H}_{2} \mathrm{O}: \mathrm{CH}_{3} \mathrm{OH}: \mathrm{NH}_{3}: \mathrm{CO}(100: 50: 10: 10)$ and (100:50:1:1). Figure adapted Bernstein et al. (1995).

cannot be explained by any of the more abundant species predicted by gas and gas-grain chemical models, but which is readily made by the radiative processing of laboratory ices containing both $\mathrm{C}$ and N. For detailed descriptions of the UV driven chemical evolution of ice, see Briggs et al. (1992), Bernstein et al. (1995), Gerakines et al. (1996) and references therein. Ultraviolet processing of the nonpolar ices of quiescent cloud regions produces $\mathrm{CO}_{2}, \mathrm{~N}_{2} \mathrm{O}, \mathrm{O}_{3}, \mathrm{CO}_{3}, \mathrm{HCO}, \mathrm{H}_{2} \mathrm{CO}$, and possibly $\mathrm{NO}$ and $\mathrm{NO}_{2}$ (Elsila et al., 1997). 
The same ice irradiation processes that produce the simpler molecules such as $\mathrm{CO}, \mathrm{CO}_{2}, \mathrm{HCO}, \mathrm{H}_{2} \mathrm{CO}, \mathrm{CH}_{4}$, etc., also produce far more complex species. The parent species and more volatile photoproducts sublime upon warm up to about $200 \mathrm{~K}$ under vacuum, leaving a mixture of less volatile species behind. The species identified in the material remaining on the window at $200 \mathrm{~K}$ and at room temperature are shown in Figure 3. These species, which result from the irradiation of laboratory ices which mimic the polar ices associated with protostellar environments differ from those produced by irradiating realistic analogs of the ices in the quiescent regions. Clear-cut evidence for these compounds in interstellar clouds is presently lacking, although some of the spectral structure detected in the $2000-1250 \mathrm{~cm}^{-1}$ region is consistent with their presence.

The residue which remains on the window at room temperature is of particular interest to the cometary community. This organic residue is rich in the cage molecule Hexamethylenetetramine (HMT, $\mathrm{C}_{6} \mathrm{H}_{12} \mathrm{~N}_{4}$ ), with lesser amounts of polyoxymethylene-related species (POMs), amides, and ketones (Bernstein et al., 1995). [As an aside, based on Bernstein et al.'s suggested HMT synthesis route which prominently invokes the reactive intermediate methyleneimine, Dickens et al. (1997) searched for this species and found it to be widespread throughout the ISM.] The dominance of HMT in photolyzed methanol-rich residues is in sharp contrast to the organic residues produced by irradiating ices which do not contain methanol (Agarwal et al., 1985), or the family of organic molecules produced in thermally promoted polymerization-type reactions in unirradiated realistic ice mixtures (Schutte et al., 1993). In non- $\mathrm{CH}_{3} \mathrm{OH}$ containing ices, HMT is only a minor product in a residue dominated by a mixture of POM related species. POMlike species have been suggested as an important organic component detected in the coma of Comet Halley (Huebner et al., 1989).

Lastly, given that PAHs are known to be widespread and abundant throughout the interstellar gas, we have studied the photochemistry of PAH-containing ices. With an abundance of $10^{-7}$ with respect to $\mathrm{H}$, PAHs are more abundant than all of the gas- phase polyatomic molecules combined (e.g. Table II). While the UV photolysis of PAHs in interstellar ices modifies only a small fraction of the interstellar PAH population, this change is significant. As shown below, the principle PAH reaction pathways induced by $\mathrm{PAH}$ photolysis in $\mathrm{H}_{2} \mathrm{O}$ ices are: hydrogenation which produces $\mathrm{H}_{n}$-PAHs; oxidation which produces ketones (quinones), alcohols, and ethers; and deuteration when $\mathrm{D}_{2} \mathrm{O}$ is present in the ice (Bernstein et al., 1996, 1999; Sandford et al., 1999). 

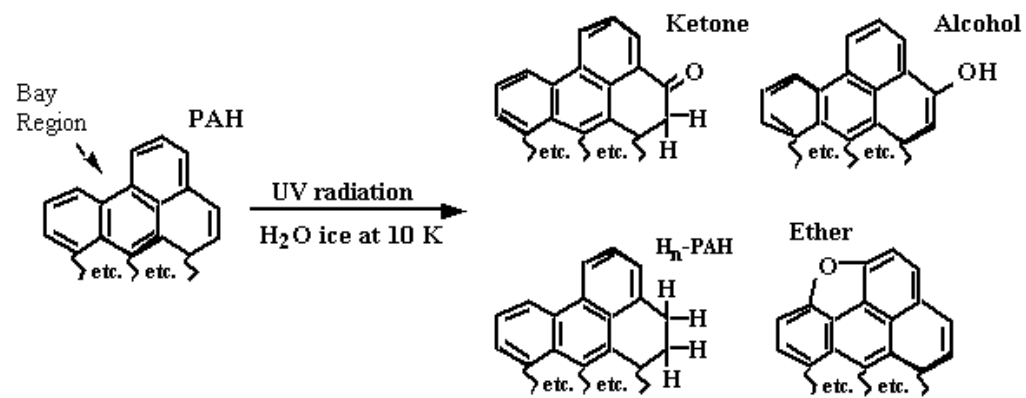

These alteration processes have dramatically different effects on the chemical nature of the parent. Hydrogen-atom addition transforms some of the edge rings into cyclic-aliphatic hydrocarbons, thereby creating molecules with both aromatic and aliphatic character and decreasing the overall degree of aromaticity. Ketone or aldehyde formation opens up an entire new range of possible chemical reactions that were impossible in the parent PAH.

These results are also important for the cometary community. First, PAH photochemistry in interstellar/pre-cometary ices is a potentially important contributor to the richness of Solar System chemistry. A strong connection of this material with the carbonaceous fractions of meteorites (and by implication comets) may well exist. For example, complex organic molecules similar to those produced here have been identified in meteorites (Hahn et al., 1988; Cronin and Chang, 1993) and oxidized polycyclic aromatic hydrocarbons are present in organic extracts from the Murchison meteorite (Deamer, 1997). Furthermore, photon-assisted PAH deuteration may bear on the deuterium enrichments of PAHs seen in meteorites (Kerridge et al., 1987) and in IDPs (Clemett et al., 1993; McKeegan et al., 1985). The formation histories of these extraterrestrial materials are not well understood, although the presence of deuterium enrichments in many of the classes of these compounds has been taken to implicate an interstellar origin (Kerridge et al., 1987; Cronin et al., 1993). Thus, the photolytic processes discussed here may well have contributed significantly to this meteoritic inventory.

\section{Conclusion}

Infrared observations, combined with realistic laboratory simulations, have revolutionized our understanding of interstellar ice and dust. These materials are of particular relevance here since these are the building blocks of comets and also because comets are thought to have brought most of the volatiles and complex organics to the early Earth. Interestingly, the similarity between the interstellar ice constituents and relative abundances with the list of known cometary constituents 
is remarkable. This strong similarity strengthens the case for applying interstellar ice studies directly to the study of comets.

Ice composition in molecular clouds depends on local conditions. In areas associated with star, planet, and comet formation, ices comprised of polar species and entrapped volatiles such as $\mathrm{H}_{2} \mathrm{O}, \mathrm{CH}_{3} \mathrm{OH}, \mathrm{CO}, \mathrm{CO}_{2}, \mathrm{NH}_{3}, \mathrm{XCN}\left(\mathrm{OCN}^{-}\right), \mathrm{H}_{2}$, and $\mathrm{H}_{2} \mathrm{CO}$ are most important. In quiescent regions, the ices are dominated by non-polar, volatile species such as $\mathrm{O}_{2}, \mathrm{~N}_{2}, \mathrm{CO}$, and $\mathrm{CO}_{2}$. Non-polar interstellar ices are far less important in the warm (by interstellar standards) star-formation regions due to the high volatility of the individual components. Photolysis of the polar, $\mathrm{H}_{2} \mathrm{O}$-rich ices produces $\mathrm{H}_{2}, \mathrm{H}_{2} \mathrm{CO}, \mathrm{CO}_{2}, \mathrm{CO}, \mathrm{CH}_{4}, \mathrm{HCO}$, and the moderately complex organic molecules: $\mathrm{CH}_{3} \mathrm{CH}_{2} \mathrm{OH}$ (ethanol), $\mathrm{HC}(=\mathrm{O}) \mathrm{NH}_{2}$ (formamide), $\mathrm{CH}_{3} \mathrm{C}(=\mathrm{O}) \mathrm{NH}_{2}$ (acetamide), $\mathrm{R}-\mathrm{CN}$ and $\mathrm{R}-\mathrm{NC}$ (nitriles and isonitriles), and hexamethylenetetramine (HMT, $\mathrm{C}_{6} \mathrm{H}_{12} \mathrm{~N}_{4}$ ), as well as more complex species including polyoxymethylene and related species (POMs), amides, and ketones. Inclusion of PAHs (also known to be ubiquitious throughout the interstellar medium) in the irradiated ices produces many species similar to those found in meteorites including aromatic alcohols, quinones and ethers. Photon assisted PAH-ice deuterium exchange also occurs. All of the above species are readily formed and thus likely cometary constituents at the 0.1 to 1 percent level.

\section{References}

Agarwal, V. K., Schutte, W., Greenberg, J. M., Ferris, J. P., Briggs, R., Connor, S., van de Bult, C. P. E. M., and Baas, F.: 1985, Origins of Life 16, 21-40.

Allamandola, L. J., and Sandford, S. A.: 1988, In Dust in the Universe (eds. M. E. Bailey and D. A. Williams), pp. 229-263. Cambridge Univ. Press, Cambridge.

Allamandola, L. J., Sandford, S. A. and Valero, G.: 1988, Icarus 76, 225-252.

Allamandola, L. J., Tielens, A. G. G. M., and Barker, J. R.: 1989, Astrophys. J. Suppl. 71, 733-755.

Allamandola, L. J., Sandford, S. A., Tielens, A. G. G. M., and Herbst, T. M.: 1992, Astrophys. J. 399, 134-146.

Altwegg, K., Balsiger, H. and Geiss, J.: 1999, Space Sci. Rev., this volume.

Bernstein, M.P., Sandford, S.A., Allamandola, L.J., Chang, S. and Scharberg, M.A.: 1995, Astrophys. J. 454, 327-344.

Bernstein, M.P., Sandford, S. A., and Allamandola, L. J.: 1996, Astrophys. J. 472, L127

Bernstein, M.P., Sandford, S.A., Allamandola, L.J., Gillette, J.S., Clemett, S.J., and Zare, R.N.: 1999, Science 283, 1135.

Blake, G.A., Sutton, E.C., Masson, C.R., and Phillips, T.G.: 1987, Astrophys. J. 315, 621.

Blake, D., Allamandola, L., Sandford, S., Hudgins, D. and Freund, F.: 1991, Science 254, 548-551.

Boogert, A.C.A., Schutte, W. A., Helmich F.P., Tielens A.G.G.M., and Wooden, D.H.: 1997, Astron. Astrophys. 317, 929,

Briggs, R., Ertem G., Ferris, J.P., Greenberg, J.M., McCain, P.J., Mendoza-Gomez, C.X., and Schutte, W.: 1992, Origins of Life and Evolution of the Biosphere 22, 287- 307

Brook, T. Y., Sellgren, K., and Geballe, T.R.: 1999, Astrophys. J. in press

Brown, P. D. and Charnley, S. B.: 1990, Mon. Not. Roy. Astron. Soc. 244, 432-443.

Capps, R.W., Gillett, F.C. and Knacke, R.F.: 1978, Astrophys. J. 226, 863-868.

Charnley, S.B., Tielens, A. G. G. M. and Millar, T. J.: 1992, Astrophys. J. 399, L71-L74. 
Chiar, J.E., Adamson, A.J., Kerr, T.H., and Whittet, D.C.B.: 1994, Astrophys. J. 426, 240.

Chiar, J.E., Adamson, A.J., Kerr, T.H. and Whittet, D. C. B.: 1995, Astrophys. J. 455, 234.

Chiar, J.E., Gerakines, P.A., Whittet, D. C. B., Pendleton, Y.J., Tielens, A.G.G.M., Adamson and Boogert, A.C.A.,: 1998, Astrophys. J. 498, 716.

Clemett, S., Maechling, C., Zare, R., Swan, P., and Walker, R.: 1993, Science 262, 721.

Cronin, J. R., Pizzarello, S., Epstein, S., and Krishnamurthy, R. V.: 1993, Geochim. Cosmochim. Acta 57, 4745.

Cronin, J.R. and Chang, S.: 1993, in The Chemistry of Life's Origins, eds. J. M. Greenberg, C. X. Mendoza-Gmez, \& V. Pirronello, (Kluwer Academic Publishers: Netherlands) p. 209

Crovisier, J. and Bockelée-Morvan, D.: 1999, Space Sci. Rev., this volume.

Deamer, D. W.: 1997, Microbiology and Molecular Biology Reviews 61, 239.

DeGrauw, et al.: 1996, Astron. Astrophys. 315, L345.

Demyk, K., Dartois, E., d'Hendecourt, L., Jourdain de Muizon, M., Heras, A., Breitfellner, M.: 1998, Astron.Astrophys. 339, 553.

d'Hendecourt, L. B., Allamandola, L. J. and Greenberg, J. M.: 1985, Astron. Astrophys. 152, 130.

d'Hendecourt, L. B. and Jordain de Muizon, M.: 1989, Astron. Astrophys. 223, L5-L8.

Dickens, J.E., Irvine, W.M., DeVries, C.H., and Ohishi, M.: 1997, Astrophys. J. 479, 307.

Ehrenfreund, P., Boogert, A.C.A., Gerakines, P.A., Schutte, W.A., Tielens, A.G.G.M., and van Dishoek, E.F.: 1996, Astron. Astrophys. 315, L341.

Ehrenfreund, P., Boogert, A.C.A., Gerakines, P.A., Tielens, A.G.G.M., and van Dishoek, E.F.: 1997, Astron. Astrophys. 328, 649.

Ehrenfreund, P., Dartois, E., Demyk, K., and d'Hendecourt, L.B.: 1998, Astron. \& Astrophys. 339, L17.

Elsila, J., Allamandola, L.J., and Sandford, S.A.: 1997, Astrophys. J.479, 818-838.

Gerakines, P. A., Schutte, W.A., and Ehrenfreund, P., 1996: Astron. Astrophys. 312, 289.

Greenberg, J. M. et al.: 1972, Mem Soc Roy Sci. de Liege 6e serie 3.

Greenberg, J. M.: 1978, In Cosmic Dust (ed. J. A. M. McDonnell), pp. 187-294. J. Wiley, New York, NY.

Greenberg, J. M. and Li, A.: 1999, Space Sci. Rev., this volume.

Grim, R.J.A., and Greenberg, J.M.: 1987a, Astrophys. J. Lett. 321, L91-L96.

Grim, R.J.A., and Greenberg, J.M.: 1987b, Astron Astrophys. 181, 155-168.

Grim, R.J.A., Baas, F., Geballe, T.R., Greenberg, J.M., and Schutte, W.A.: 1991, Astron. Astrophys. 243, 473-477.

Hagen, W., Allamandola, L. J. and Greenberg, J. M.: 1980, Astron. Astrophys. 86, L3-L6.

Hahn, J. H., Zenobi, R., Bada, J. L., and Zare, R. N.: 1988, Science 239, 1523.

Huebner, W.F., Boice, D.C., and Korth, A.: 1989, Adv. Space Res. 9, 29.

Irvine, W. M.: 1999, Space Sci. Rev., this volume.

Kerridge, J. F., Chang, S., and Shipp, R., 1987: Geochim. Cosmochim. Acta 51, 2527.

Lacy, J. H., Baas, F., Allamandola, L. J., Persson, S. E., McGregor, P. J., Lonsdale, C. J., Geballe, T. R. and van der Bult, C. E. P.: 1984, Astrophys. J. 276, 533-543.

Lacy, J., Carr, J., Evans, N., Baas, F., Achtermann, J. and Arens, J.: 1991, Astrophys. J. 376, 556-560.

Lacy, J., Faraji, H., Sandford, S.A., and Allamandola, L.J.: 1998, Astophys. J. 501, L105.

Leger, A., Gauthier, S., Defourneau, D. and Rouan, D.: 1983, Astron. Astrophys. 117, 164-169.

McKeegan, K. D., Walker, R. M., and Zinner, E.: 1985, Geochim. Cosmochim. Acta 49, 1971.

Moore, M. H., Donn, B., Khanna, R. and A'Hearn, M. F.: 1983, Icarus 54, 388-405.

Moore, M. H. and Hudson, R.: 1992, Astrophys. J. 401, 353-360.

Mumma, M.J., Stern, S.A., and Weissman, P.R.: 1993, in Protostars and Planets III, eds E.H. Levy, J.I. Lunine, , and M.S. Matthews, (University of Arizona Press, Tucson), 1177.

Ohishi, M., Irvine, W. M., and Kaifu, N.: 1992, in Astrochemistry of Cosmic Phenomena, ed. P.D. Singh, (Kluwer, Dordrecht), 171. 
Omont, A., Moseley, S. H., Forveille, T., Glaccum, W. J., Harvey, P. M., Likkel, L., Loewenstein, R. F., and Lisse, C. M.: 1990, Astrophys. J. 355, L27-L30.

Palumbo, M. E., Tielens, A. G. G. M., and Tokunaga, A. T.: 1995, Astrophys. J. 449, 674-680.

Puget, J. L. and Leger, A.: 1989, Ann. Rev. Astron. Astrophys. 27, 161-198.

Reuter, D. C.: 1992, Astrophys. J. 386, 330-335.

Sandford, S. A., Allamandola, L. J., Tielens, A. G. G. M. and Valero, G.: 1988, Astrophys. J. 329, 498-510.

Sandford, S. A., Allamandola, L. J. and Geballe, T. R.: 1993, Science 262, 400-402.

Sandford, S. and Allamandola, L. J.: 1993a, Astrophys. J. (Letters) 409, L65-L68.

Sandford, S. A. and Allamandola, L. J.: 1993b, Astrophys. J. 417, 815-825.

Sandford, S.A.: 1996, Meteoritics \& Planetary Science 31, 449-476.

Sandford, S. A., Bernstein, M. P., Allamandola, L. J., Gillette, J. S., and Zare, R. N.: 1999, Astrophys. $J .$, in press.

Schutte, W. A., Allamandola, L. J. and Sandford, S. A.: 1993, Icarus 104, 118-137.

Schutte, W. A.: 1996, In The Cosmic Dust Connection (ed. J. M. Greenberg), D. Reidel, Dordrecht, TheNetherlands, p. 1.

Schutte, W.A., Gerakines, P.A., Geballe, T.R., van Dishoek, E.F., and Greenberg, J.M.: 1996, Astron Astrophys. 309, 633-647.

Schutte, W.A. and Greenberg, J.M.: 1997, Astron. \& Astrophys. 317, L43.

Smith, R. G., Sellgren, K. and Tokunaga, A. T.: 1989, Astrophys. J. 344, 413-426.

Smith, R. G., Sellgren, K. and Brooke, T. Y.: 1993, Mon. Not. Roy. Astron. Soc. 263, 749-766.

Tegler, S. C., Weintraub, D. A., Allamandola, L. J., Sandford, S. A., Rettig, T. W. and Campins, H.: 1993, Astrophys. J. 411, 260-265.

Tegler, S. C., Weintraub, D. A., Rettig, T. W., Pendleton, Y. J., Whittet, D. C. B. and Kulesa, C. A.: 1995, Astrophys. J. 439, 279- 287.

Tielens, A. G. G. M. and Hagen, W.: 1982, Astron. Astrophys. 114, 245-260.

Tielens, A. G. G. M., Allamandola, L. J., Bregman, J., Goebel, J., d'Hendecourt, L. B. and Witteborn, F. C.: 1984, Astrophys. J. 287, 697-706.

Tielens, A. G. G. M. and Allamandola, L. J.: 1987, In Physical Processes in Interstellar Clouds (eds. G. E. Morfill and M. Scholer), pp. 333-376. D. Reidel, Dordrecht, The Netherlands

Tielens, A. G. G. M., Tokunaga, A. T., Geballe, T. R. and Baas, F.: 1991, Astrophys. J. 381, 181

van Dishoek et al.:1996, Astron. Astrophys. 315, L349

Willner, S. P.: 1977, Astrophys. J. 214, 706-711.

Winnewisser, G. and Kramer, C.: 1999, Space Sci. Rev., this volume.

Address for correspondence: Louis J. Allamandola, lallamandola@mail.arc.nasa.gov 